\title{
Continuous exposure of pancreatic cancer cells to dietary bioactive agents does not induce drug resistance unlike chemotherapy
}

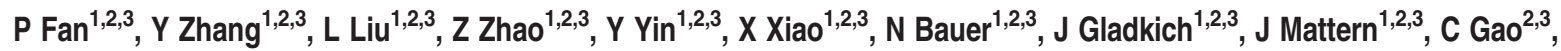 \\ P Schemmer ${ }^{2,3}$, W Gross ${ }^{1,2,3}$ and I Herr, ${ }^{*, 1,2,3}$
}

The repeated treatment of cancer cells with chemo- or radiotherapy induces therapy resistance, but it was previously unknown whether the same effect occurs upon continuous exposure of cancer cells to diet-derived chemopreventive agents. We elucidated this interesting question in pancreatic ductal adenocarcinoma, which is a highly aggressive cancer entity with a marked resistance toward gemcitabine and other cytotoxic drugs. The isothiocyanate sulforaphane, present in cruciferous vegetables, and the polyphenol quercetin, present in many fruits and vegetables induced apoptosis and reduced viability in gemcitabine-sensitive BxPC-3 cells but not in non-malignant ductal pancreas cells and mesenchymal stromal cells. In turn, BxPC-3 cells were treated with increasing concentrations of gemcitabine, sulforaphane or quercetin for more than 1 year and the surviving subclones Bx-GEM, Bx-SF and Bx-Q were selected, respectively. While Bx-GEM cells acquired a total resistance, Bx-SF or Bx-Q cells largely kept their sensitivity as proved by MTT assay, annexin staining and FACS analysis. The evaluation of the self-renewal-, differentiation- and migration-potential by colony formation, differentiation or migration assays demonstrated that cancer stem cell features were enriched in gemcitabine-resistant cells, but decreased in sulforaphane- and quercetin-long time-treated cells. These results were confirmed by orthotopic xenotransplantation of cancer cells to the mouse pancreas, where Bx-GEM formed large, Bx-Q small and Bx-SF cells almost undetectable tumors. An mRNA expression profiling array and subsequent gene set enrichment analysis and qRT-PCR confirmed that tumor progression markers were enriched in Bx-GEM, but reduced in Bx-SF and Bx-Q cells. This study demonstrates that the continuous exposure of pancreatic cancer cells to sulforaphane or quercetin does not induce resistance in surviving cells but reduces tumorigenicity by inhibition of tumor progression markers. These results highlight that cancer cells may not adapt to the preventive and therapeutic effects of a regular fruit- and vegetable-based diet.

Cell Death and Disease (2016) 7, e2246; doi:10.1038/cddis.2016.157; published online 2 June 2016

Pancreatic ductal adenocarcinoma (PDA) is a highly aggressive malignancy, which is reflected by it's tenth place of estimated new cancer cases per year, but it's fourth place of estimated cancer deaths in males. ${ }^{1}$ Surgical resection is the only potentially curative therapy, but merely $15-20 \%$ of tumors are resectable, due to early metastasis, missing early symptoms and late diagnosis. ${ }^{2}$ Gemcitabine is considered as standard chemotherapy in PDA treatment, despite a low rate of responsiveness due to a marked resistance to chemoand radiotherapy. ${ }^{3}$ The newer combination chemotherapy FOLFIRINOX extends life by 4 months when compared with gemcitabine but has more side effects. ${ }^{4}$

Chemoresistance, either acquired or intrinsic, is a major limitation in the successful treatment of pancreatic cancer. The frequent application of chemotherapy to cancer patients is due to the observation that it often succeeds in reducing a tumor mass and improves survival. However, the transition of the cancer to a resistant stage, called acquired resistance, is a key factor for the failure of chemotherapeutic agents. ${ }^{5}$ Recently, the high intrinsic resistance of pancreatic cancer was associated with a high basal percentage of the otherwise small amount of cancer stem cells (CSCs). ${ }^{6}$ Also, tumor progression was associated with the enrichment of CSCs, for example, of $\mathrm{PDA}^{7}$ that survive anti-proliferative chemotherapeutics and contribute to disease progression. ${ }^{8}$

CSCs are considered to possess 'stemness' like normal stem cells including an enhanced tumor initiating potential, and the ability to tumorigenicity, self-renewal, differentiation and migration. ${ }^{9,10}$ Various dysregulated signaling pathways have an important role in maintaining the stemness character of CSCs including self-renewal, epithelial-mesenchymal transition (EMT) and others. ${ }^{11}$ In solid tumors, chemotherapyresistant CSCs were commonly detected, for example, in cancer of the breast, ${ }^{12}$ colorectum, ${ }^{13}$ prostate, ${ }^{14}$ ovary, ${ }^{15}$ lung, ${ }^{16}$ liver, ${ }^{17}$ glioblastoma, ${ }^{18}$ osteosarcoma ${ }^{19}$ and PDA. ${ }^{20}$ In particular, the enrichment of CSCs and drug resistance was found in PDA after repeated treatment with gemcitabine. ${ }^{21}$

Several epidemiological studies suggest that cancer development and progression are possibly correlated to a defined dietary pattern. Silverman et al. ${ }^{22}$ found in a large population-

\footnotetext{
${ }^{1}$ Molecular OncoSurgery, University of Heidelberg, Heidelberg, Germany; ${ }^{2}$ Section Surgical Research, University of Heidelberg, Heidelberg, Germany and ${ }^{3}$ Department of General, Visceral and Transplantation Surgery, University of Heidelberg, Heidelberg, Germany

*Corresponding author: I Herr, Section Surgical Research, Department of General Surgery, University of Heidelberg, Im Neuenheimer Feld 365, Heidelberg 69120, Germany. Fax: +49 622156 6402; E-mail: i.herr@ uni-heidelberg.de 
based case-control study that the consumption of cruciferous vegetables, for example, broccoli, cauliflower and cabbage, three and more times weekly, reduced the risk of pancreatic cancer by about $50 \%$ and therefore could have a preventive role. The main bioactive substance from broccoli and cauliflower is the mustard oil and isothiocyanate sulforaphane. Besides, the antioxidative polyphenol quercetin is present in broccoli and other cabbage varieties, but also in many other fruits and vegetables, such as berries, onions and apples. ${ }^{23}$ Several laboratory and animal studies exist and suggest that sulforaphane and quercetin inhibit proliferation and metastasis and enhance apoptosis and eliminate CSC features in pancreatic cancer. Therefore, these bioactive agents are considered as promising future treatment options. ${ }^{24-29}$

The question is whether a future therapeutic treatment with sulforaphane or quercetin may induce drug resistance after frequent exposure, as known for chemotherapy. In the present study, we showed that frequently repeated cycles of sulforaphane and quercetin exposure did not induce drug resistance but reduced the tumorigenic potential and the expression of progression markers. In contrast, continuous exposure to gemcitabine induced a total drug resistance along with enhanced tumorigenicity.

\section{Results}

Quercetin and sulforaphane selectively reduce the viability in malignant cells. To establish the optimal dose response of PDA cell lines to quercetin and sulforaphane, the established gemcitabine-sensitive PDA cell line BxPC-3 was used, along with non-malignant primary human pancreatic duct cells (CRL-4023) and human mesenchymal stromal cells, which served as controls. After treatment with different concentrations of quercetin or sulforaphane, the cell morphology and viability were determined $72 \mathrm{~h}$ later by microscopy and MTT assay. The number of BxPC-3 cells strongly decreased after treatment and apoptotic blebbing appeared (Figure 1a). Quercetin and sulforaphane significantly inhibited the viability of BxPC-3 cells in a dose-dependent manner (Figure 1b). In contrast, different quercetin concentrations did not reduce the viability of the non-malignant cell line CRL-4023. Likewise, only high SF doses slightly affected the viability of CRL-4023 cells at $72 \mathrm{~h}$. Similarly, quercetin or sulforaphane only weakly reduced the viability of mesenchymal stromal cells (Figure 1d). The DMSO solvent alone, at a dilution of $1: 1000$, which was the lowest concentration of dilution of the DMSO quercetin or sulforaphane stock in medium, did not affect the cell viability (Figures $1 \mathrm{~b}-\mathrm{d}$ ), as expected.

\footnotetext{
Selection of BxPC-3 subclones by continuous exposure to gemcitabine, sulforaphane or quercetin. To compare the effects of long-time treatment with gemcitabine, quercetin and sulforaphane, the K-Ras wildtype and gemcitabinesensitive PDA cell line BxPC-3 was chosen to enable the selection of resistant subclones by continuous long-time treatment. Thus, BxPC-3 cells were exposed to continuously higher concentrations of gemcitabine, quercetin or sulforaphane for more than 1 year (Figure 2a). The final surviving
}
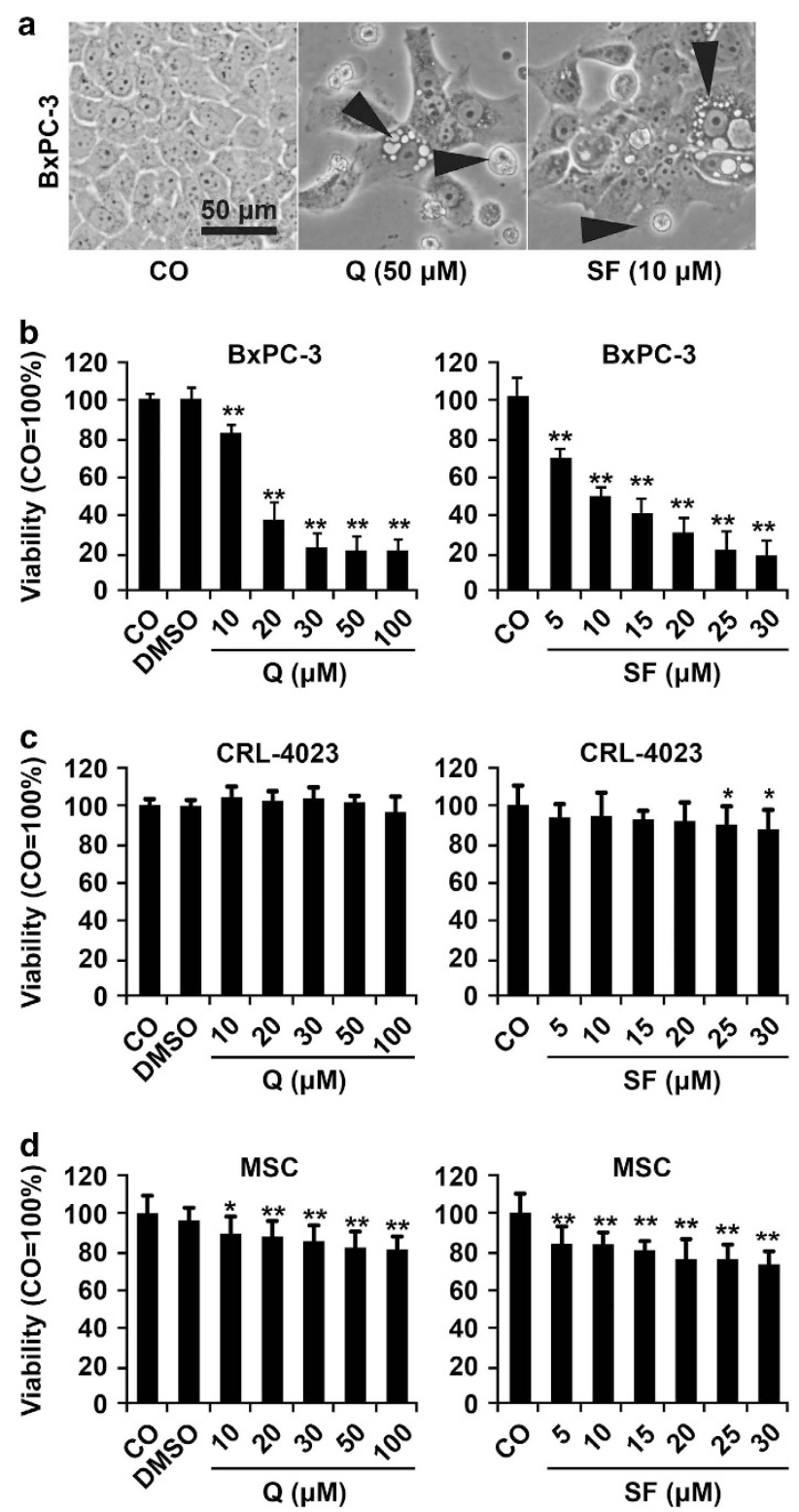

Figure 1 Quercetin and sulforaphane are selectively cytotoxic in pancreatic cancer cells. (a) The human PDA cell line BxPC-3 was left untreated (CO) or was treated with quercetin $(\mathrm{Q}, 50 \mu \mathrm{M})$ or sulforaphane (SF, $10 \mu \mathrm{M})$, followed by microscopy and photography $48 \mathrm{~h}$ later. Representative pictures at $\times 200$ magnification are shown. (b) Cell viability was determined by MTT assay in untreated BxPC-3 cells (CO) or $72 \mathrm{~h}$ after treatment with vehicle alone diluted $1: 1000$ (DMSO) or with increasing concentrations of quercetin $(\mathrm{Q})$ from 5 to $100 \mu \mathrm{M}$ and sulforaphane (SF) from 5 to $30 \mu \mathrm{M}$, diluted in DMSO whose final concentration in medium was $1: 1000$ or higher. (c) The non-malignant primary human cell lines CRL-4023 (immortalized ductal pancreas cells) or (d) MSC (bone marrow-derived) were treated with different concentrations of quercetin (Q) or sulforaphane (SF) as indicated, while DMSO (1: 1000) was set as a control. The cell viability was measured as described above. Three independent experiments were performed at least in triplicates and the data are presented as means \pm S.D. ${ }^{*} P<0.05,{ }^{* *} P<0.01$

subclones were selected and named Bx-GEM, Bx-Q and $B x-S F$. To determine whether the subclones acquired resistance toward the respective treatments, they were left untreated for 2 weeks and then treated again. The percentages of 


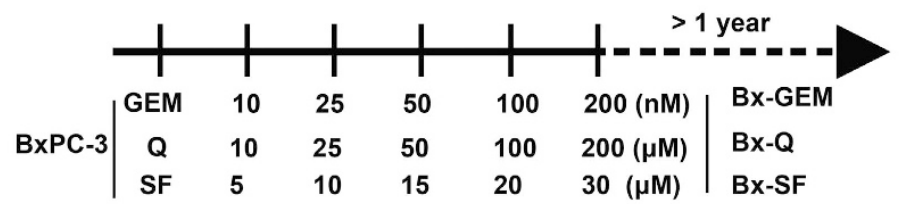

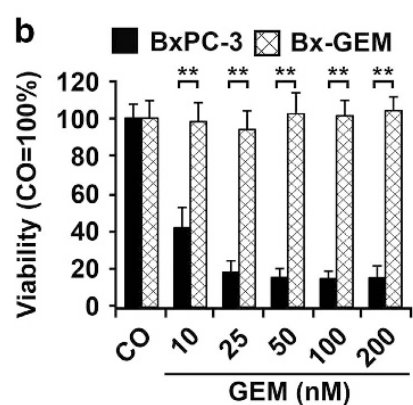
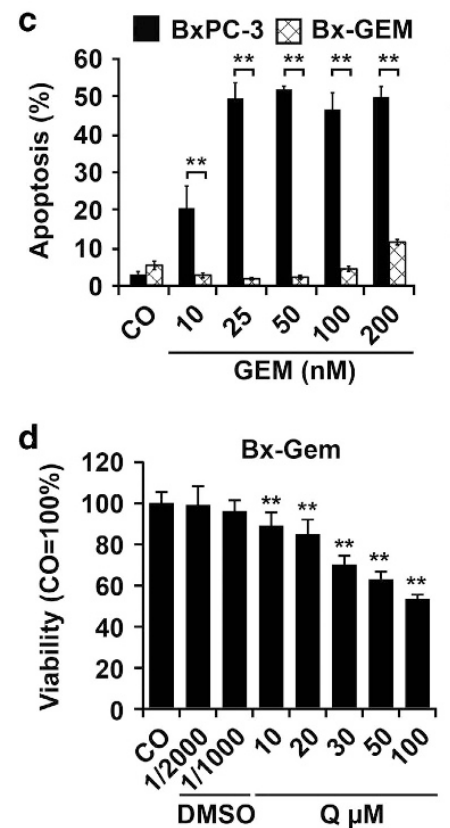

BxPC-3 $\square$ Bx-Q

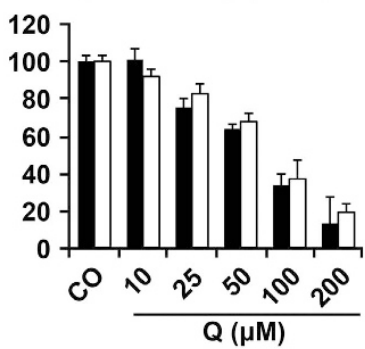

BxPC-3 $\square$ Bx-Q

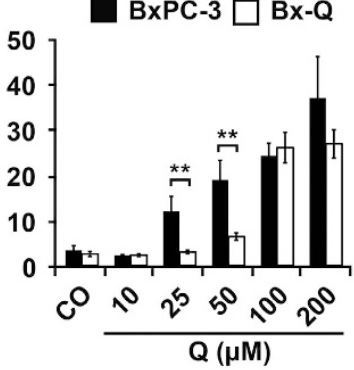

BxPC-3 $\square$ Bx-SF
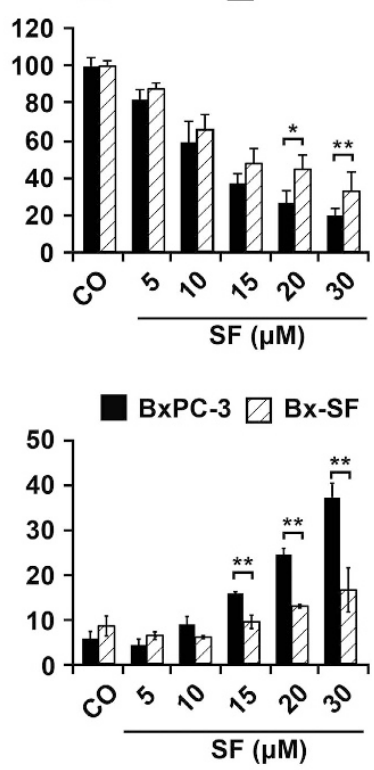

Figure 2 Continuous exposure to gemcitabine but not to sulforaphane or quercetin leads to pronounced therapy resistance. (a) BxPC-3 cells were treated with gemcitabine (GEM, $10 \mathrm{nM})$, quercetin $(\mathrm{Q}, 10 \mu \mathrm{M})$ or sulforaphane $(\mathrm{SF}, 5 \mu \mathrm{M})$ at a confluence of $40-60 \%$. After 2 weeks, when the cells recovered, they were treated again with higher concentrations of each agent as indicated, followed by a recovery phase and a new round of treatment with higher concentrations. After more than 1 year of repeated treatment with gradually higher concentrations, the highest concentrations of $200 \mathrm{nM} \mathrm{GEM}, 200 \mu \mathrm{M} \mathrm{Q}$ and $30 \mu \mathrm{M}$ SF were reached and the resulting final subclones were named Bx-GEM, $B x-Q$ and Bx-SF, respectively. These final subclones were used for all following experiments. (b) The cells were treated with different concentrations of gemcitabine, quercetin or sulforaphane as indicated. Seventy-two hours later, the viability was measured by MTT assay. (c) Likewise, the percentage of apoptotic cells was measured by staining with Annexin V-FITC and PI followed by FACS analysis. (d) Bx-GEM cells were treated with quercetin or sulforaphane at concentrations indicated; and $72 \mathrm{~h}$ later, the viability was measured by MTT assay. Three independent experiments were performed for (b-d) (MTT assay $n=8$, apoptosis assay $n=3$ ) and the data are presented as means \pm S.D. ${ }^{\star} P<0.05,{ }^{*} P<0.01$

viability and apoptosis were determined $72 \mathrm{~h}$ later by MTT assay (Figure $2 \mathrm{~b}$ ) or by staining with Annexin V-FITC and PI, followed by FACS analysis (Figure 2c). Although Bx-GEM cells were totally resistant to gemcitabine, even with the highest concentration of $200 \mathrm{nM}$, as we confirmed in our recent study, ${ }^{30}$ the treatment of $B x-Q$ cells with quercetin or of Bx-SF cells with sulforaphane still significantly reduced the viability and enhanced apoptosis. However, both $B x-Q$ and Bx-SF subclones were slightly more resistant to quercetin or sulforaphane, compared with parental BxPC-3 cells. Most importantly, totally gemcitabine-resistant Bx-GEM cells were sensitive to quercetin or sulforaphane, as concluded from a reduced viability after treatment (Figure 2d). To examine whether a change in the genetics of Bx-GEM cells might have been the reason for the gemcitabine resistance, we forwarded them together with parental BxPC-3 cells to a commercially available Multiplex Cell Line Authentication Test. In the resulting report, both BxPC-3 and Bx-GEM cells were confirmed to be $100 \%$ identical with the original BxPC-3 cells in the database (data not shown), suggesting that the resistance may be due to the upregulation of resistance mechanisms. 
Continuous quercetin and sulforaphane exposure reduces tumorigenicity in vitro. To investigate CSC features, we evaluated the colony formation, migration and differentiation capacities of parental BxPC-3 cells and their subclones. The cells were treated and 3 days later an equal number of surviving cells were seeded for colony formation. Ten days later, this resulted in a significantly enhanced number of colonies in Bx-GEM cells, but in a lower number in Bx-Q and Bx-SF cells compared with parental BxPC-3 cells (Figure 3a). To evaluate the long-lasting effect of treatment, an equal number of surviving cells from colonies of each group were selected, followed by re-seeding and detection of second-generation colonies 2 weeks later. While Bx-GEM cells still exhibited a high colony-forming capacity, Bx-Q and Bx-SF cells had a low to nearly absent colony-forming capacity. To elucidate the invasion potential, a scratch assay was performed. A confluent cell layer was wounded with the tip of a 10- $\mu$ l pipette; and the closure of the wounded region was evaluated by microscopy $24 \mathrm{~h}$ later. Whereas the gap was totally closed in Bx-GEM cells, it was still slightly open in BxPC-3 cells and widely open in Bx-Q and Bx-SF cells (Figure $3 b$ ). Then, the differentiation potential was tested by culturing the cells in osteogenic differentiation medium for 14 days, followed by staining with SIGMAFAST BCIP/NBT (Sigma-Aldrich, St. Louis, MO, USA) substrate for detection of alkaline phosphatase produced by cells differentiated into osteoblasts. We found that the Bx-GEM cells differentiated with a high potential, whereas the differentiation potential of $B x-Q$ and Bx-SF cells was reduced compared with parental BxPC-3 cells (Figure 3c). To further define cancer progression markers, we detected the expression of the CSCs surface marker EpCAM (also known as ESA), the selfrenewal marker Nanog, the mesenchymal cell marker Twist2 and the epithelial cell marker E-cadherin by western blot analysis (Figure $3 d$ ). Whereas the expression of EpCAM, Nanog and Twist2 was almost reduced in Bx-Q and Bx-SF cells, E-cadherin was induced, but the opposite occurred in Bx-GEM cells, compared with parental BxPC-3 cells. Therefore, continuous exposure to gemcitabine strongly enhanced progression markers, whereas long-time treatment with quercetin or sulforaphane reduced them.

Continuous quercetin and sulforaphane exposure reduces tumorigenicity in vivo. To further compare the tumorigenicity between parental BxPC-3 cells and the derived subclones in vivo, an equal number of each cell line was orthotopically transplanted to the pancreas of immunodeficient mice. Six weeks later, the mice were killed and the xenograft tumors resected, followed by the measurement of the tumor volumes. Compared with the parental cells, the tumor volume significantly increased in mice harboring Bx-GEM xenografts, but it was reduced in mice with $B x-Q$ tumors and almost not detectable in mice with Bx-SF xenografts (Figure 4a). To evaluate the expression of proliferation marker Ki67, we performed immunofluorescence staining followed by microscopy (Figure 4b). Whereas Bx-GEM cells had a slightly higher, but not significantly different proliferation rate compared with BxPC-3 cells, the proliferation was significantly decreased in $B x-Q$ xenografts and further diminished in tumors derived from Bx-SF cells. Because the expression of the proliferation marker Ki67 did not significantly differ between xenografts of parental BxPC-3 and Bx-GEM cells, we wondered whether the larger tumor volume of Bx-GEM cells might be due to a reduced basal apoptosis. Thus, we stained the cleaved fragment of active caspase-3 in xenograft tissue and evaluated the percentage of positive cells in 10 vision fields. Though the percentage of apoptosis was lower in Bx-GEM-derived xenografts compared with BxPC-3-derived tumors, this difference was not statistically significant (Supplementary Figure 1).

Continuous quercetin and sulforaphane exposure reduces gene array-analyzed expression of progression markers. To investigate CSC features on the mRNA expression level, total RNA from parental BxPC-3 cells and the derived final subclones Bx-GEM, Bx-Q and Bx-SF was extracted; and an mRNA profiling analysis was performed from triplicates by the use of the Human HT-12 v4 Expression Bead Chip Kit with 44052 genes. The relative expression of significantly changed genes $(P<0.01)$ is shown in a heat map (Figure $5 \mathrm{a}$ ). The number of differentially expressed mRNAs in parental cells compared with the derived three subclones is presented as a Venn diagram (Figure 5b). A comparison of gene expression between Bx-GEM, Bx-Q and Bx-SF cells revealed the differential regulation of 2513 genes. Among them, 1192 genes were differentially regulated between Bx-GEM and Bx-Q cells, 1534 between Bx-GEM and Bx-SF cells and 1164 genes between $B x-Q$ and Bx-SF cells. Compared with parental BxPC-3 cells, 9235, 5972 and 6742 genes were differentially regulated in Bx-GEM, Bx-Q and BxSF cells, respectively (Figure $5 \mathrm{c}$ ). Next, we selected those candidate genes, which were most significantly differentially regulated and related to CSCs, by chemoresistance or EMT by the use of the Ingenuity Pathway Analysis (IPA) computer program. The expression patterns of the predicted candidate genes were confirmed by qRT-PCR (Figure $5 d$ ). Interferon alpha-inducible protein 27 (IFI27), which confers EMT, tumorigenicity and stemness, ${ }^{31}$ stromal interaction molecule 1 (STIM1), which promotes tumor metastasis ${ }^{32}$ and apoptosis resistance, ${ }^{33}$ melanoma antigen family B2 (MAGEB2), which activates cell proliferation and resistance to ribotoxic stress, ${ }^{34}$ and kinase anchor protein 12 (AKAP12), which promotes tumor development and metastasis, ${ }^{35}$ were all upregulated in $\mathrm{Bx}$ GEM cells but downregulated in Bx-Q and Bx-SF cells compared with parental BxPC-3 cells.

Continuous quercetin and sulforaphane exposure reduces the expression of progression markers. To characterize the gene array results by an additional computational method, we performed a gene set enrichment analysis (GSEA) to identify those differentially regulated genes typical for drug resistance and stemness. The GSEA computational method determines whether an a priori defined set of genes shows statistically significant, concordant differences between two biological states (http://www.broadinstitute.org/gsea/index. jsp), or in our case, between parental BxPC-3 cells and the derived subclones Bx-GEM, Bx-Q or Bx-SF. We used the ready-to-use KESHELAVA_MULTIPLE_DRUG_RESISTANCE set, which includes 88 genes related to chemoresistance and the RAMALHO_STEMNESS_UP set, which includes 206 genes, known to be enriched in embryonic, neural and 

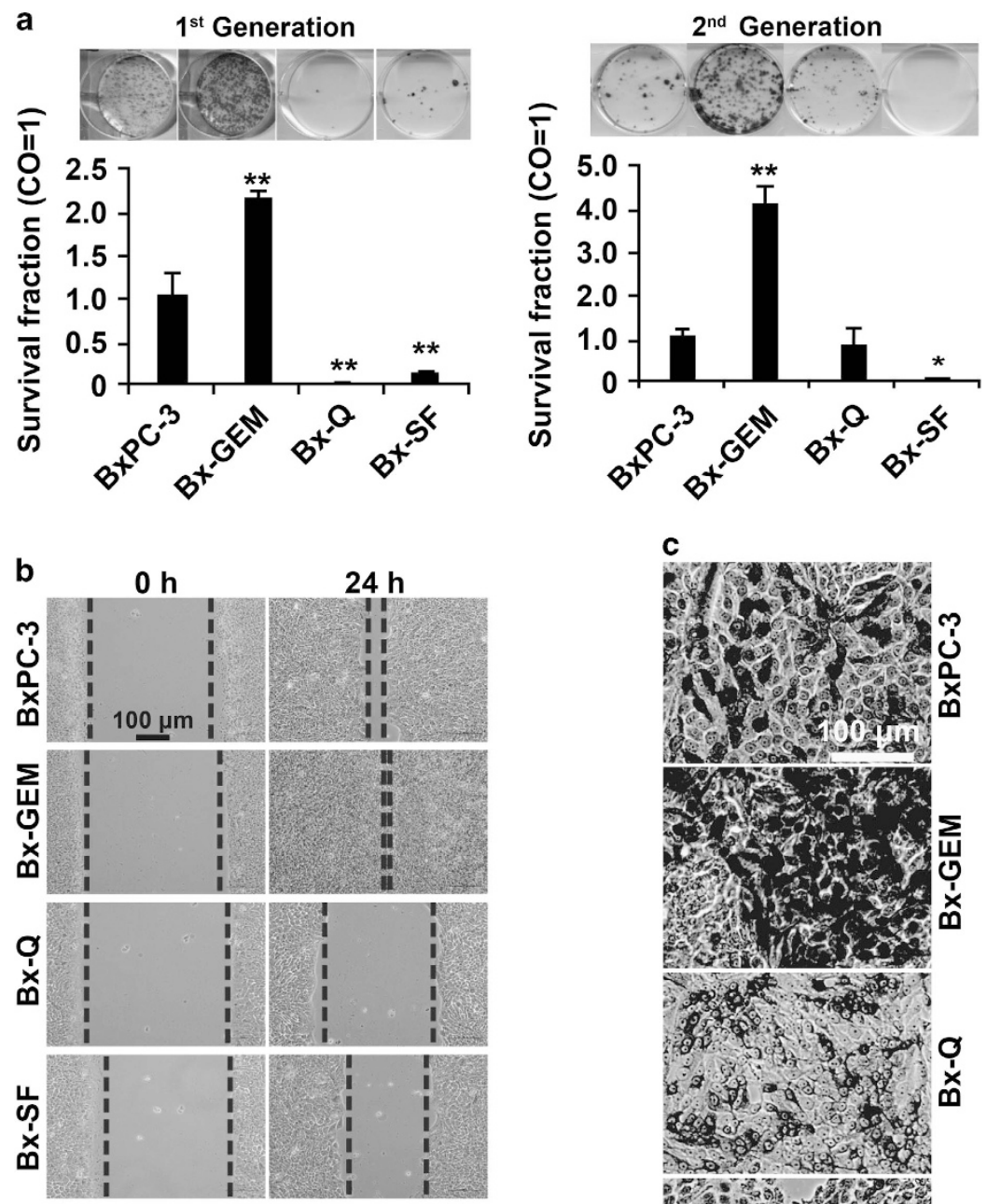

C
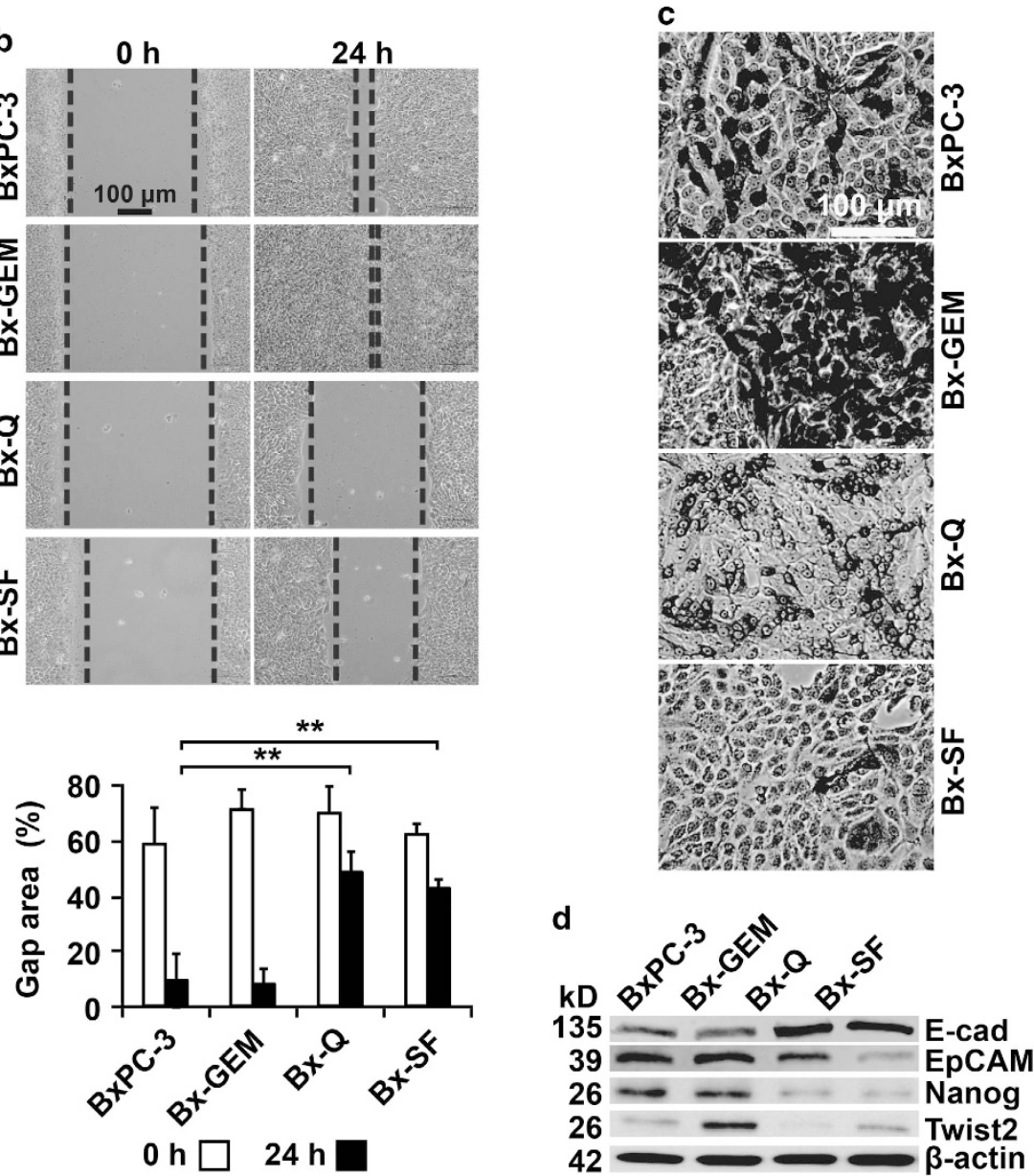

Figure 3 Continuous exposure to gemcitabine increases tumorigenicity but continuous exposure to sulforaphane or quercetin reduces it. (a) BxPC-3, Bx-GEM, Bx-Q and BxSF cells were seeded at a low density (2000 cells/well) in 6-well plates. After 2 weeks, cells were Coomassie-stained and colonies containing more than 50 cells were counted under a dissecting microscope. The survival fraction and representative photographs of colonies (first generation) are presented on the left. For second-generation colony formation, an equal amount of living cells from first-generation colonies were collected and 2000 cells per well were re-seeded. The colony formation was analyzed as described above and is presented on the right. (b) Cells were cultured to $90 \%$ confluence before the cell layer was scratched with the tip of a 10- $\mu$ l pipette. Closure of the wounded region was evaluated $24 \mathrm{~h}$ after scratching by microscopy at $\times 100$ magnification. For quantification of the scratched area, the percentage of the gap area was evaluated and calculated by TScratch software (diagram below photographs). (c) Cells were seeded in 6-well plates, followed by exposure to NH Osteo-Diff medium to induce osteocytic differentiation. Fourteen days later, the cells were stained with BCIP/NBT substrate for alkaline phosphatases, expressed by cells differentiated into osteocytes, which appear dark. Representative images at $\times 200$ magnification are shown. (d) Proteins were harvested and the expression of EpCAM, Nanog, Twist2 and E-cadherin was measured by western blot analysis. $\beta$-Actin was used as a loading control. Three independent experiments were performed at least in triplicates and the data are presented as means \pm S.D. ${ }^{*} P<0.05$, ${ }^{* \star} P<0.01$ 

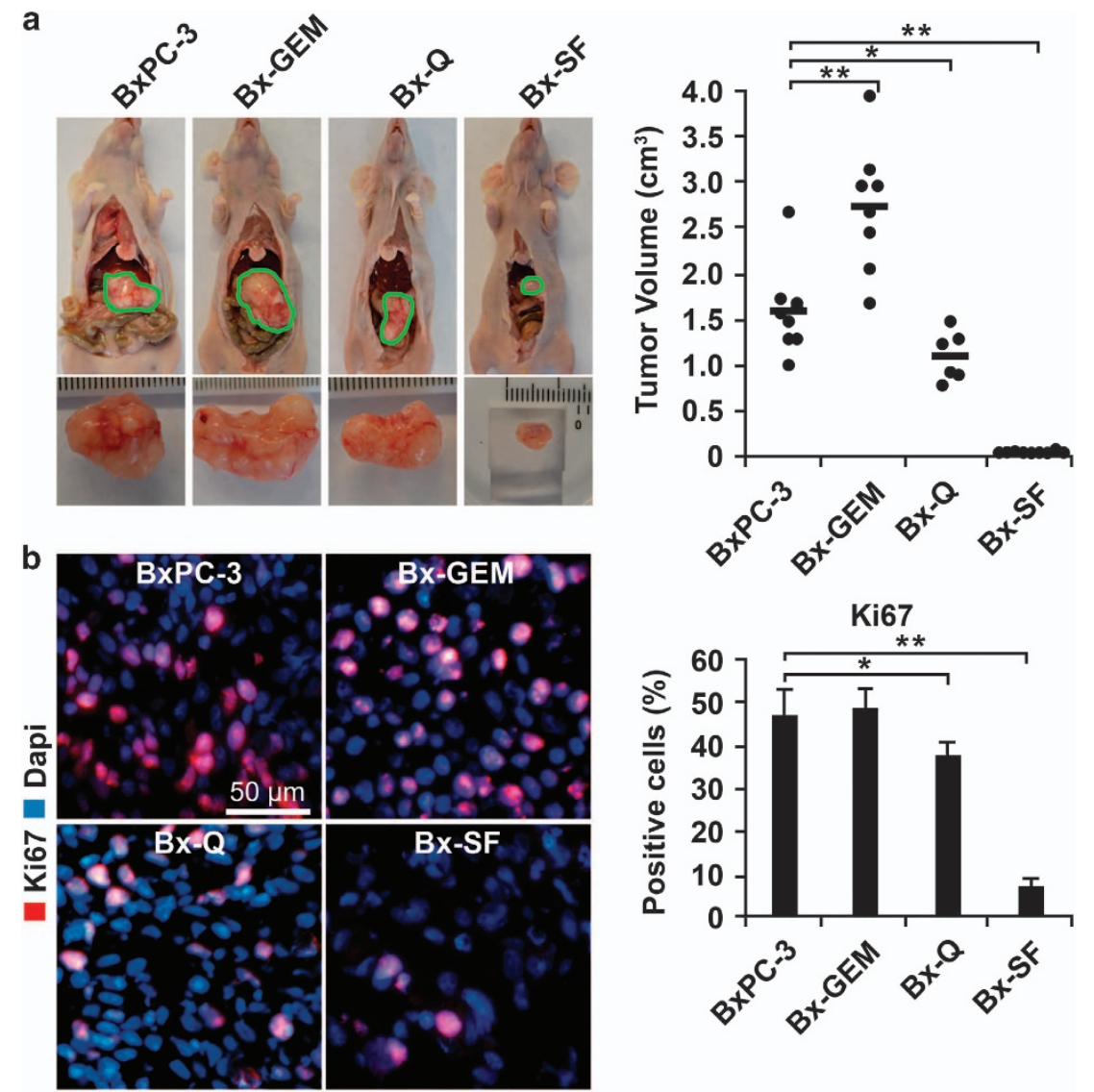

Figure 4 Continuous exposure to quercetin or sulforaphane inhibits tumorigenicity in vivo, whereas gemcitabine-treatment enhances it. (a) Immunodeficient mice $(n=8 / p e r$ cell line) were anesthetized, followed by surgical intervention to expose the pancreas and to inject $1 \times 10^{5}$ cells in $10 \mu$ l matrigel into the pancreatic head. After closure of the wound, the mice were kept for 6 weeks to allow tumor development, followed by euthanasia, tumor resection and measurement of tumor volumes by calipers. Representative images of tumor xenografts from each group are shown on the left and a diagram with the individual tumor volumes and the means of each group \pm S.D. on the right. (b) Tumor tissue sections from xenografts were evaluated by immunofluorescence staining for the expression of the proliferation marker Ki67 and representative pictures at $\times 200$ magnification are shown on the left. The percentage of positive cells was counted and the means \pm S.D. are shown in the diagram on the left. ${ }^{*} P<0.05,{ }^{* *} P<0.01$

hematopoietic stem cells (compare Supplementary Table 1). ${ }^{21}$ Regarding the expression of multidrug-resistance genes, Bx-Q and Bx-SF cells showed no significant changes compared with parental BxPC-3 cells, but Bx-GEM cells had a significant upregulation (Figure 6a). The detailed differential expression of each gene is shown in the heat map (Figure 6b). For instance, FBX011, which served as an oncogene in breast cancer and was related to chemoresistance, ${ }^{36}$ was significantly upregulated in Bx-GEM cells compared with BxPC-3 cells. Likewise, Bx-GEM cells exhibited a significant enrichment of genes related to stemness features, whereas Bx-SF cells had a significantly decreased expression compared with parental BxPC- 3 cells and no significant change was found in $\mathrm{Bx}-\mathrm{Q}$ cells (Figures $7 a$ and $b$ ). For example, compared with BxPC-3, Bx-SF exhibited a lower level expression of the YES-YAPTEAD2 signaling pathway, which was reported to maintain embryonic stem cell self-renewal. ${ }^{37}$

\section{Discussion}

In the present study, the situation of acquired therapy resistance was mimicked by long-time treatment of gemcitabine-sensitive BxPC-3 cells with continuously increasing concentrations of gemcitabine for more than 1 year, to select the drug-resistant, final population, as described. ${ }^{38}$ In addition, we investigated the important question, whether pancreatic cancer can acquire resistance by the continuous exposure to sulforaphane or quercetin, resembling a long-time fruit- and vegetable-enriched diet. Whereas the gemcitabine-selected clone Bx-GEM was totally resistant toward gemcitabine, it was still sensitive to quercetin and sulforaphane. Likewise, long-time quercetinor sulforaphane-treated $\mathrm{Bx}-\mathrm{Q}$ or $\mathrm{Bx}-\mathrm{SF}$ cells were only slightly resistant and almost kept their sensitivity toward the respective stimulus. Although most of the long-time sulforaphane- or quercetin-treated BxPC-3 cells underwent apoptosis after each new round of sulforaphane or quercetin treatment, a small percentage of cells survived. The reason for this slight adaption to these bioactive agents is unclear, but we found that it is not due to the activation of conventional resistance mechanisms. Accordingly, $\mathrm{Bx}-\mathrm{Q}$ and Bx-SF cells had a lower colony- and spheroidforming capacity and migrated slower compared with parental BxPC-3 cells. In contrast, these tumorigenic features were 
a
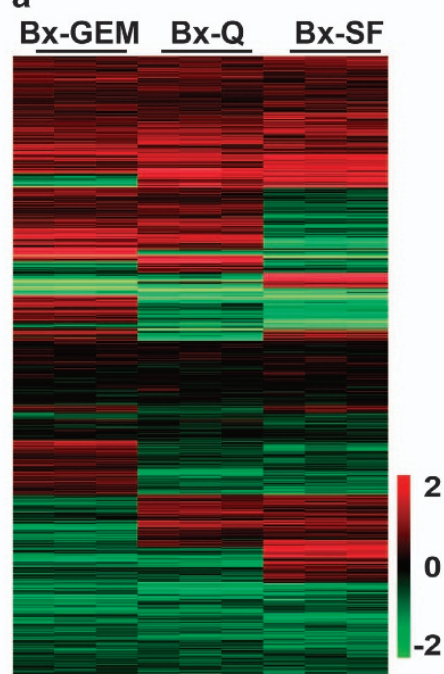

b

b BX-GEM BX-Q

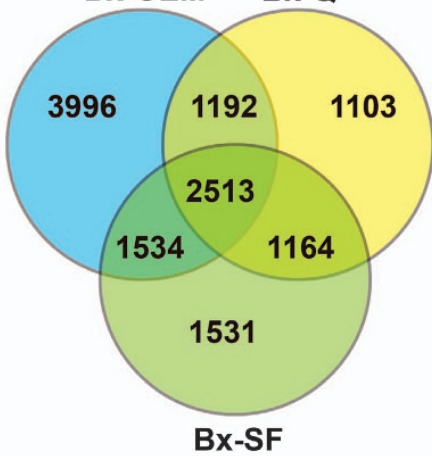

C

mRNA profiling results

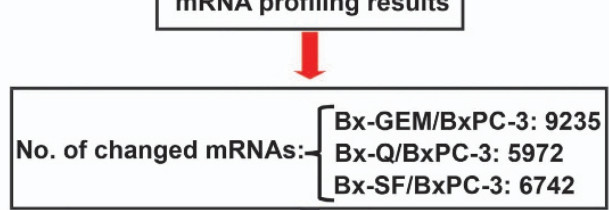

Selection of most significantly differentially regulated genes between BxPC-3/Bx-GEM, BxPC-3/Bx-Q and BxPC-3/Bx-SF

BX-GEM/BXPC-3

$\mathrm{BX}-\mathrm{Q} / \mathrm{BxPC}-3$

Bx-SF/BxPC-3

\begin{tabular}{|l|l|}
\hline $\begin{array}{l}\text { STIM1 } \\
\text { PRKCDBP }\end{array}$ & \\
IFI27 & GAGE7 \\
HISTIH4H & \\
\hline
\end{tabular}

\begin{tabular}{|l|l|}
\hline RAB31 & SH3PXP2A \\
IFI27 & KYNU \\
MAGEB2 & TCN1 \\
SLC43A3 & \\
GALNTL4 & \\
\hline
\end{tabular}

\begin{tabular}{|l|l|}
\hline TUBB6 & \\
AKAP12 & TCEA3 \\
RAB31 & \\
& \\
\hline
\end{tabular}

Selection of genes related to

CSCs, EMT, chemoresistance

BX-GEM/BxPC-3

Bx-Q/BxPC-3

IFI27, MAGEB2

\begin{tabular}{c} 
Bx-SF/BxPC-3 \\
\hline AKAP12 \\
\hline
\end{tabular}
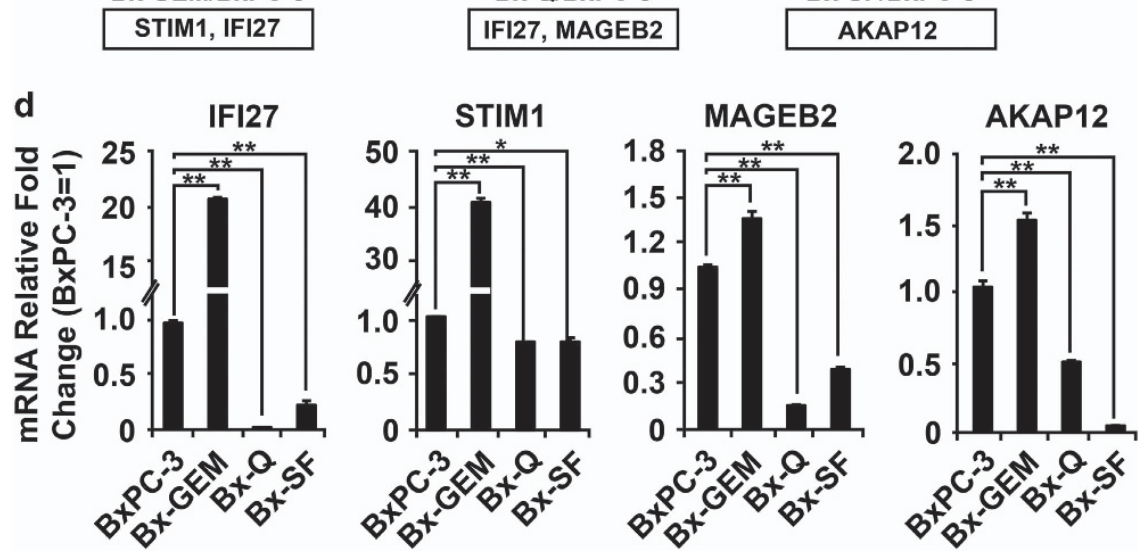

Figure 5 Gene array analysis demonstrates differentially regulated genes after continuous exposure to sulforaphane, quercetin or gemcitabine. (a) mRNA was harvested from BxPC-3, Bx-GEM, Bx-Q and Bx-SF cells followed by gene array analysis and biostatistical evaluation of statistically significant expression patterns between the different cell lines. The heat map shows the relative expression of differentially regulated genes in Bx-GEM, Bx-Q and Bx-SF cells compared with parental BxPC-3 cells. (b) The numbers of differentially regulated genes in Bx-GEM, Bx-Q and Bx-SF cells compared with parental BxPC-3 cells and compared between the groups are presented in a Venn diagram $(P<0.01)$. (c) Flow diagram of the computational selection process of miRNA candidates from the miRNA profiling results is shown above. Upward blue arrows: upregulation of mRNAs compared with BxPC-3; Downward blue arrows: downregulation of mRNAs compared with BxPC-3. (d) RNA was harvested from BxPC-3, Bx-GEM, Bx-Q and Bx-SF cells; and mRNA expression of IFI27, STIM1, MAGEB2 and AKAP12 was analyzed by qRT-PCR. The expression in BxPC-3 cells was set to 1 . GAPDH was used as an endogenous control. The qRT-PCR was performed in triplicates three times with similar outcome; and the means \pm S.D. are shown 
a
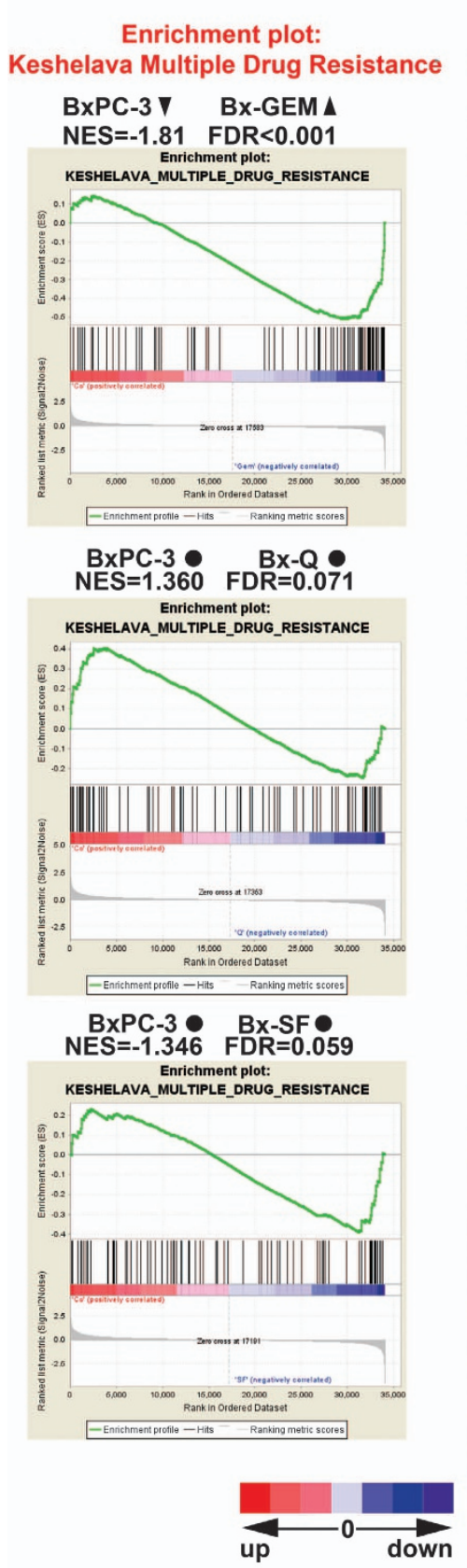

b

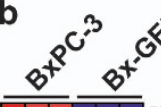

$8^{+0^{20}}$

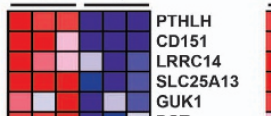

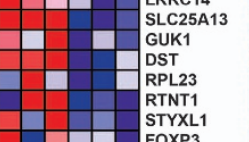

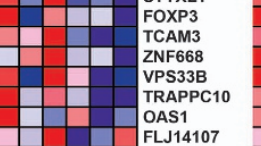

KTAA1609

TBSP

TP53TG1
MY01F

GGA2

TMEM104

MARCH2

KLRF1
HGSNAT

MAN1B1

BCL7B

CCDC109B

STL1

TSPAN8
PPP4C

TBX1

CCHCR1

MTAP
ANXA2P2

ANXX2P2
PEX10

TNPP5B
DNALTJ1

DNALTJ1
EGOT

EGOT

HDAC1
KCTD17

KCTD17

NDRG2
CYTH1

CYTH1
TMED10

HFE
PDLJM5

PDLJM5
HHAT

MAPK12

DOM 32

ACADS

DOCK9
TQGAP1

USE1

PHF1
CCS

ODF2

BRE

TAF12

UFC1
PCTP

HSPB1

SBNO2

MED8
NXT2

ENC1

MAGOHB

SLC48A1

TSPAN31

BCAP31

BLVRB

CCDC92

CASP4
CDKN2C

CDKN2C
SEPX1

ADAM15

FBX011
HTST2H2A

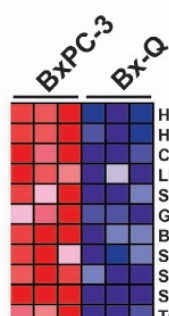

0

$8^{8^{c^{3}}} 8^{x^{5^{x}}}$

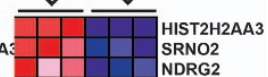

NDRG2
KTAA160

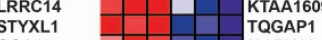

$\begin{array}{lll}\text { GGA2 } & \\ \text { MAPK12 }\end{array}$

$\begin{array}{lll}\text { BCL7B } & \text { LRRC14 } \\ \text { SBNO2 } & \text { SLC48A1 }\end{array}$

SLC25A13

TCAM3

PTHLH

NDRG2

1
DOCK9
DOCKF

ZNF668

ENC1

MED8

MAGOH

RTNT1

CYTH1

KTAA1609
FLJ14107

FLJ14107

RPL23

KCTD17

DNALJ1
OAS1

TMED10

TMED10
PEX10

PEX10
TRAPPC10

KLRF1

FN1

UFC1

ADAM15

TQGAP1

CCDC92
TP53TG1

TP53TG1
TBX1

HGSNAT

MTAP

HFE

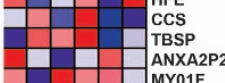

ANXA2P
MY01F

USE1

ACADS

TMEM104

SEPX1

VPS33B

FOXP3

CCHCR1

EGOT

PDLJM5

MAPK12

ODF2 2

CCDC109B

CD151
MARCH2

PPP4C

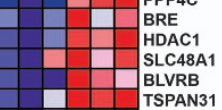

BLVRB
TSPAN31

GUK1

MAN1B1

CRTP2
TAF12

DST

BCAP31

CASP4
NXT2

NXT2
GSTK1

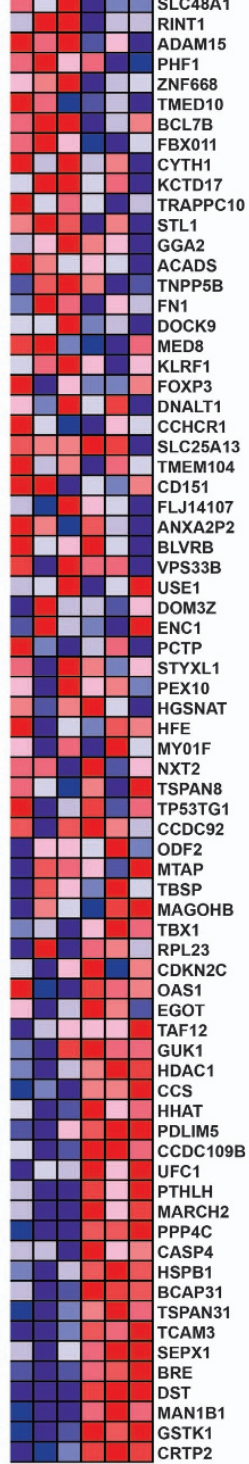

Figure 6 Gene set enrichment analysis demonstrates the induction of multidrug resistance in Bx-GEM but not in Bx-Q and Bx-SF cells. (a) Significantly differentially regulated genes from the gene array analysis described in Figure 5 were further analyzed by a gene set enrichment analysis with an available set of genes known to be involved in multidrug resistance (KESHELAVA_MULTIPLE_DRUG_RESISTANCE). NES, normalized enrichment score; FDR, false discovery rate. $\boldsymbol{\Delta}$ Enriched. No change. $\nabla$ Decreased. The differences were considered significant if FDR values were less than 0.05 . The detailed process is described in Materials and Methods. (b) Heat map of the differentially regulated mRNAs according to gene set enrichment analysis as described in (a). The scale marks the relative changes of differentially regulated mRNAs in BxPC-3, Bx-GEM, Bx-Q and BxSF: upregulated (red), no change (gray) and downregulated (blue)

enhanced in Bx-GEM. Our data suggest that an enhanced self-renewal potential was rather not the reason for the observed slight apoptosis resistance of $\mathrm{Bx}-\mathrm{Q}$ cells to quercetin and of Bx-SF cells to sulforaphane. In support of this hypothesis, the examination of the differentiation potential and the protein expression of EMT and stemness markers revealed an enhanced differentiation potential in Bx-GEM, but a reduced potential in Bx-SF and Bx-Q cells. Therefore, we assume that $\mathrm{Bx}-\mathrm{Q}$ and $\mathrm{Bx}-\mathrm{SF}$ cells adapted somehow to quercetin or sulforaphane treatment, although this adaption is not due to enhanced tumorigenicity and the underlying mechanism requires further investigation. 
a

a Enrichment plot:

Ramalho-Stemness up

BXPC-3 \ BX-GEM A

NES $=-1.38$ FDR=0.045

Enrichment plot: RAMALHO_STEMNESS_UP

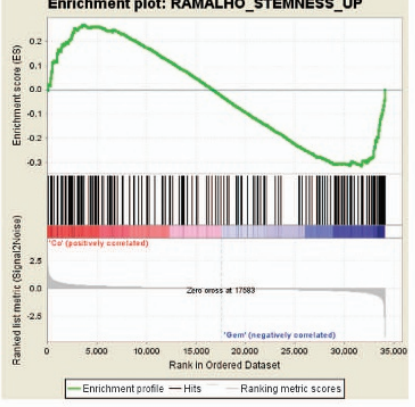

BxPC-3 $\quad B x-Q$

NES $=-1.23 \quad F D R=0.073$

Enrichment plot: RAMALHO_STEMNESS_UP

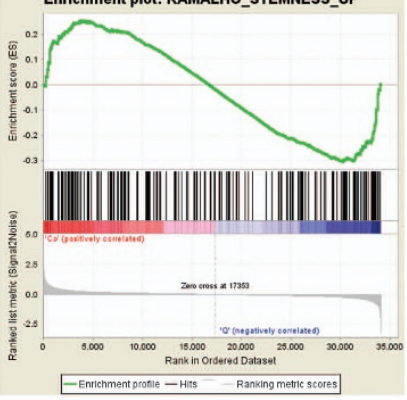

BXPC-3 BX-SF V

NES $=1.56$ FDR $<0.001$

Enrichment plot: RAMALHO_STEMNESS_UP
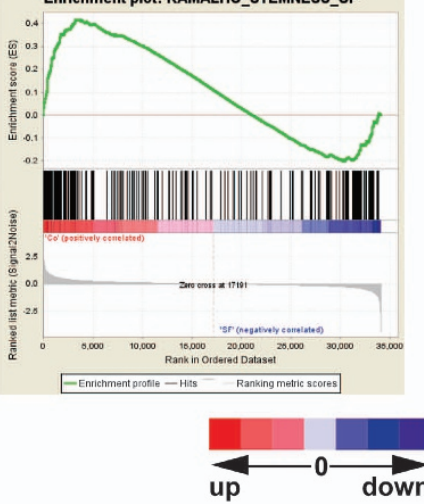

b

$s^{c_{3}^{3}}$

sर $^{+\infty}$

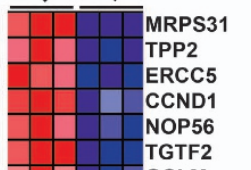

TGTF2

GCLM

GNL2

GARS

STXBP3

TEAD2

CAD

LAPTM4B

NOP58

PTPN2

WDR43

KRAS

CTBP2

TNP0

BYSL

PSMD11

MPDU1

ARCN1

DPH5

TNTS5

PDCD2

STAM

GRWD1

CDKN1A

STRN3

NDUFAF1

TARS

RYK

TTGA6

MED23

KIF2A

MDFIC

TMEM1

RNF4

RCL1

FHL1

CRR3
PKD2

CDK2AP1

FBX08

UBE2T

TMPAD1

PLS3

PEX7

TTGB1

TARS2

CRTAP

RASA1

RAD23B

CPXM1

SLC38A2

ALDH7A1

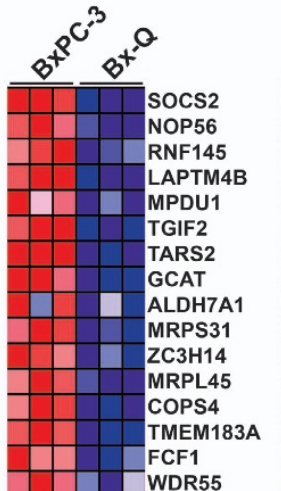

$8^{x^{c^{3}}} s^{x^{x^{5}}}$

TEAD2

NOP58

UPP1
WDR43

CDKN1A

PTPN2

CAD

CAD

LJMA1

YES1

ZMAT3

TARS

TARS

HSPA4

GCLM

ELOVL6

SLC4A7

NOP56

SLC38A2

GRWD1

GRWD1
CPXM1

TNPO1

RCN1

RCN1

YWHAH

RSL1D1

PPP2R1B

GNL2

GNL2

RPUSD4

MRPS10

UTP20

ADAM 9

HRSP12

SOCS2

ZNF213

ZNF639

EPRS

NDUFAF1

RYK

FBXO8

FBXO8
PPTC

TXNRD1

SNX12

RPL22

$\mathrm{DPH} 2$

XRCC5

XPOT

TARS2

STRN3

SH3D19

SH3D19

SUCLG2

PEXT

DCTPP1

TMEM183A

TRC1D15

CRTAP

CRTAP

ACAT2

UBE2T

WBP5

Figure 7 Gene set enrichment analysis demonstrates the induction of stemness genes in Bx-GEM but reduction in Bx-SF and no change in Bx-Q cells. (a) Significantly differentially regulated genes from the gene array analysis described in Figure 5 were further analyzed by a gene set enrichment analysis with an available set of genes known to be involved in stemness (RAMALHO_STEMNESS_UP) and further analyzed as described in Figure 6a. (b) Heat map of the differentially regulated mRNAs according to gene set enrichment analysis as described in (a)

These in vitro data were further underlined by orthotopic xenotransplantation to the mouse pancreas, which resulted in a significantly reduced tumor volume after transplantation of Bx-SF or BX-Q cells, whereas Bx-GEM cells formed much larger tumors compared with parental BxPC-3 cells. In correspondence to the colony-forming potential in vitro, tumors derived from Bx-SF cells even smaller or totally absent than those derived from $B x-Q$ cells. Interestingly, although the means of Ki67-positive cells in tumor tissue were higher in $\mathrm{Bx}-\mathrm{GEM}$ and lower in Bx-Q and Bx-SF cells compared with
BxPC-3 cells, and vice versa for the cleaved fragment of active caspase-3, these differences were not statistically relevant. Therefore, we assume that the observed higher tumor volume of Bx-GEM-derived xenografts may be due to a slightly higher proliferation rate and a slightly lower basal apoptosis rate, which cannot be proved by the rather insensitive method of immunofluorescence staining and counting the number of positive cells by visual inspection.

To get knowledge about differential gene expression, which might be responsible for the observed effects, we performed 
mRNA profiling, selected differentially regulated candidate genes linked to EMT, CSC and chemoresistance signaling and confirmed their expression by qRT-PCR. In addition, a GSEA was used to compare the gene difference between parental cells and the derived subclones. Although the candidates identified by both selection methods were not identical, we demonstrated that multidrug resistance- and stemnessassociated genes were enriched in Bx-GEM cells but not in $B x-Q$ and Bx-SF cells. Most excitingly, stemness-related genes were even decreased in Bx-SF cells.

In the clinical settings, it is important to achieve a therapeutically sufficient plasma concentration of quercetin or sulforaphane. The plasma concentration typically found in humans after consumption of foods containing flavonoids is between 0.06 and $7.6 \mu \mathrm{M}$, which corresponds to recently detected plasma concentrations of quercetin. ${ }^{39}$ Peak plasma concentrations of $108.7 \pm 41.67 \mu \mathrm{M}$ quercetin have been observed; ${ }^{40}$ and a tolerance of quercetin doses ranging from $60 \mathrm{mg} / \mathrm{m}^{2} \quad(=1.5 \mathrm{mg} / \mathrm{kg})$ to $1700 \mathrm{mg} / \mathrm{m}^{2} \quad(=42.5 \mathrm{mg} / \mathrm{kg})$ is known. ${ }^{41}$ However, the therapeutic active doses of quercetin are difficult to be realized by nutrition alone. For example, to achieve about $500 \mathrm{mg}$ of quercetin, this would amount to the consumption of $1 \mathrm{~L}$ of red wine $(19 \mathrm{mg}), 1 \mathrm{~kg}$ apples $(140 \mathrm{mg}), 1 \mathrm{~kg}$ yellow onions $(347 \mathrm{mg})$ or $1 \mathrm{~kg}$ broccoli (30 mg). ${ }^{27}$ Regarding sulforaphane intake, data from a prospective Canadian epidemiological study suggest that a high consumption of broccoli or cauliflower at almost one serving per week, while three to five servings were more effective, is associated with inhibition of metastasis in prostate cancer. $^{42}$ Experimental sulforaphane concentrations, which inhibited growth of human pancreatic cancer xenografts on mice, were $4.4 \mathrm{mg} / \mathrm{kg}$ per day. ${ }^{24}$ Extrapolating this experimental concentration to humans suggests a dose of $0.36 \mathrm{mg} / \mathrm{kg}$ according to the body surface area normalization method. ${ }^{43}$ This corresponds to $25 \mathrm{mg} / 70 \mathrm{~kg}$ human, which is hard to be reached by eating mature broccoli florets alone. However, an alternative to consumption of mature broccoli for high intake of sulforaphane may be the intake of special freeze-dried broccoli sprouts or broccoli seed extract preparations. Such sprouts, seeds and derived extracts are available from several manufacturers, and they usually contain about 10-100 times more glucoraphanin, on a weight basis, than mature broccoli florets. ${ }^{44}$ Therefore, to achieve an effective concentration, supplements with high concentrations of quercetin or sulforaphane may improve the clinical outcome of chemotherapy.

In conclusion, our results highlight that a frequent consumption of quercetin or sulforaphane supplements may have preventive and therapeutic effects in pancreatic cancer.

\footnotetext{
Materials and Methods

Human primary and established cell lines. The human established pancreatic cancer cell line BxPC-3 and the human hTERT-HPNE immortalized pancreatic ductal cell line CRL-4023 were obtained from ATCC and cultured in DMEM supplemented with 10\% FCS and $5 \%$ HEPES or in ATCC complete growth medium, respectively. Mesenchymal stromal/stem cells (MSCs) were isolated from bone marrow and cultured as described. ${ }^{45}$ All cells were grown in a humidified incubator at $37^{\circ} \mathrm{C}$ and $5 \% \mathrm{CO}_{2}$. BxPC-3 cells were recently authenticated by a commercial service (Multiplexion, Heidelberg, Germany). Mycoplasma-negative cultures were ensured by monthly mycoplasma tests.
}

Reagents. Gemcitabine solution (Eli Lilly, Indianapolis, IN, USA) was obtained from the Pharmacy of the University Clinic of Heidelberg at a concentration of $76 \mathrm{mg} / \mathrm{ml}$ and stored at $4{ }^{\circ} \mathrm{C}$. This stock was freshly diluted in DMEM to a $100-\mu \mathrm{M}$ stock. Quercetin ( $\geq 95 \%)$ and sulforaphane ( $\geq 95 \%)$ (Sigma-Aldrich, St. Louis, MO, USA) were dissolved in DMSO to stock concentrations of $200 \mathrm{mM}$ or $100 \mathrm{mM}$, respectively, and stored in aliquots at $-20^{\circ} \mathrm{C}$. Each stock was used only once immediately after thawing. The final concentrations of the solvents in media were $0.1 \%$ or less.

Viability assay. The viability was measured using 3-(4,5-dimethylthiazol-2-yl)-2, 5-diphenyltetrazolium bromide (MTT) as described. ${ }^{24}$

Measurement of apoptosis. The cells were stained with FITC-conjugated Annexin V (BD Biosciences, Heidelberg, Germany) and propidium iodide $(5 \mathrm{mg} / \mathrm{ml})$ after respective treatments and analyzed by flow cytometry (Guava EasyCyte, Cytometer, Millipore, Darmstadt, Germany), as described. ${ }^{24}$

Colony formation assay. Cells were seeded at a low density in complete medium in 6-well tissue culture plates. Colonies were evaluated 10-14 days later as described. $^{24}$

Differentiation assay. Cells were seeded in 6-well plates. After $24 \mathrm{~h}$, the medium was replaced by $2 \mathrm{ml}$ osteogenic differentiation medium. The cells were cultured for 10 days. Differentiated cells were detected and images of stained cells were taken as described. ${ }^{46}$

Wound healing assay. Cells $\left(5 \times 10^{5}\right)$ were seeded in 6-well plates and grown to confluence. A line was then scraped within the confluent cells using a $10-\mu l$ pipette tip. Images of the wound healing area were acquired by microscopy $24 \mathrm{~h}$ later as described; and the area was quantified by TScratch software as described. ${ }^{47}$

Western blot analysis. Protein extracts were prepared by standard protocols and proteins were detected by western blot analysis as described. ${ }^{24}$ Antibodies were mouse monoclonal anti-EpCAM (HEA125), ${ }^{48}$ anti-Twist2 (Abcam, Cambridge, UK), anti-Nanog and anti- $\beta$-actin (Sigma-Aldrich) and rabbit monoclonal anti-E-cadherin (24E10, Cell Signalling Technology, Danvers, MA, USA).

Immunofluorescence staining. Immunofluorescence staining of samples from tumor xenografts was performed as described. ${ }^{24}$ Mouse monoclonal antibodies against Ki67 (Abcam) were used.

mRNA profiling. The RNeasy Mini Kit was used to extract total RNA according to the instructions of the manufacturer (Qiagen, Hilden, Germany); and the expression profiling was performed at the Genomics and Proteomics Core Facility of the German Cancer Research Center (DKFZ) Heidelberg, using the Human HT-12 v4 Expression Bead Chip Kit.

qRT-PCR. The RNA concentrations were measured with a NanoDrop 2000 Spectrophotometer (Nano Drop Technologies, Wilmington, DE, USA); and $500 \mathrm{ng}$ total RNA was reverse transcribed to cDNA using the High Capacity RNA to cDNA Kit (Thermo Fisher Scientific GmbH, Dreieich, Germany). Real-time PCR was performed using the Taqman Gene Expression master mix (Thermo Fisher Scientific). Primers for IFI27, STIM1, AKAP12, MAGEB2 and GAPDH were obtained from Thermo Fisher Scientific.

Bioinformatics. GSEA was performed using the GSEA version 2.2.1 from the Broad Institute at MIT. The gene sets of KESHELAVA_MULTIPLE_DRUG_RESISTANCE and RAMALHO_STEMNESS_up were used. With 1000 permutations, the cutoff of the significance level was chosen as $P<0.05$. The GSEA is described in detail at http://software.broadinstitute.org/gsea/doc/GSEAUserGuideFrame.html.

Nude mice and xenografts. A total of $1 \times 10^{5}$ cells in $10 \mu \mathrm{l}$ matrigel were injected into the subscapular region near to the head of the pancreas of 6-week-old $\mathrm{NMRI}(\mathrm{nu} / \mathrm{nu}$ ) male mice (day 0 ) through an abdominal midline incision with a fine needle after the mouse was under general anesthesia. To avoid possible leakage of tumor cells, the injection was performed under a Leica M651 microscope (Leica, Wetzlar, Germany). The mice were killed 6 weeks after tumor transplantation and 
the tumor volumes $(V)$ were measured by two diameters and calculated using the formula $V=1 / 2\left(\right.$ length $\times$ width $\left.^{2}\right)$. Animal experiments were performed in the animal facilities of the University of Heidelberg after receiving approval from the authorities (Regierungspräsidium Karlsruhe, Germany).

Statistical evaluations. Data obtained with established cell lines are presented as the means \pm S.D. from at least three separate experiments, each performed at least in triplicate. The mouse experiment was performed twice with statistically sufficient group sizes. The significance of the data was analyzed with Student's $t$-test for parametric data and the Mann-Whitney test with Bonferroni corrections for non-parametric data. $P<0.05$ was considered as statistically significant $\left({ }^{* *} P<0.01,{ }^{*} P<0.05\right)$.

\section{Conflict of Interest}

The authors declare no conflict of interest.

Acknowledgements. We thank Dr. S Karakhanova for critical text revision and Dr. F Schönsiegel, A Abukiwan and C Nwaeburu for helpful discussion. This study was supported by grants from the Federal Ministry of Education and Research (BMBF 031A213), Foundation of Surgery Heidelberg, Foundation for Cancer- and Scarlet-Research, Dietmar Hopp-Foundation and the Hanns A. Pielenz-Foundation.

\section{Author contributions}

IH contributed to concept and design. PF, YZ, LL, ZZ, NB, JG and YY contributed to acquisition of data. PF, YZ, IH, LL and YY contributed to analysis and interpretation of data. IH, PF and LL contributed to writing, review, revision of the manuscript. WG contributed to statistics.

1. DeSantis CE, Lin CC, Mariotto AB, Siegel RL, Stein KD, Kramer JL et al. Cancer treatment and survivorship statistics, 2014. CA Cancer J Clin 2014; 64: 252-271.

2. Konstantinidis IT, Warshaw AL, Allen JN, Blaszkowsky LS, Castillo CF, Deshpande V et al. Pancreatic ductal adenocarcinoma: is there a survival difference for $\mathrm{R} 1$ resections versus locally advanced unresectable tumors? What is a "true" R0 resection? Ann Surg 2013; 257: 731-736.

3. Gukovskaya AS, Pandol SJ. Cell death pathways in pancreatitis and pancreatic cancer. Pancreatology 2004; 4: 567-586

4. Conroy T, Desseigne F, Ychou M, Bouche O, Guimbaud R, Becouarn Y et al. FOLFIRINOX versus gemcitabine for metastatic pancreatic cancer. N Engl J Med 2011; 364: 1817-1825.

5. Vidal SJ, Rodriguez-Bravo V, Galsky M, Cordon-Cardo C, Domingo-Domenech J. Targeting cancer stem cells to suppress acquired chemotherapy resistance. Oncogene 2014; 33: 4451-4463.

6. Adikrisna R, Tanaka S, Muramatsu S, Aihara A, Ban D, Ochiai T et al. Identification of pancreatic cancer stem cells and selective toxicity of chemotherapeutic agents. Gastroenterology 2012; 143: e237.

7. Chuthapisith S. Cancer stem cells and chemoresistance. In: Shostak PS (ed). Cancer Stem Cells Theories and Practice. Available from: http://www.intechopen.com/books/cancer-stemcells-theories-and-practice/cancer-stem-cells-and-chemoresistance. InTech, 2011.

8. Dick JE. Stem cell concepts renew cancer research. Blood 2008; 112: 4793-4807.

9. Visvader JE, Lindeman GJ. Cancer stem cells in solid tumours: accumulating evidence and unresolved questions. Nat Rev Cancer 2008; 8: 755-768.

10. Akhtar K, Bussen W, Scott SP. Cancer stem cells - from initiation to elimination, how far have we reached? (Review). Int J Oncol 2009; 34: 1491-1503.

11. Chen $\mathrm{K}$, Huang $\mathrm{YH}$, Chen $\mathrm{JL}$. Understanding and targeting cancer stem cells: therapeutic implications and challenges. Acta Pharmacol Sin 2013; 34: 732-740.

12. Shafee N, Smith CR, Wei SZ, Kim Y, Mills GB, Hortobagyi GN et al. Cancer stem cells contribute to cisplatin resistance in Brca1/p53-mediated mouse mammary tumors. Cancer Res 2008; 68: 3243-3250.

13. Cammareri P, Scopelliti A, Todaro M, Eterno V, Francescangeli F, Moyer MP et al. Aurora-A is essential for the tumorigenic capacity and chemoresistance of colorectal cancer stem cells. Cancer Res 2010; 70: 4655-4665.

14. Liu T, Xu FH, Du XL, Lai DM, Liu TJ, Zhao YR et al. Establishment and characterization of multi-drug resistant, prostate carcinoma-initiating stem-like cells from human prostate cancer cell lines 22RV1. Mol Cell Biochem 2010; 340: 265-273.

15. Hu L, McArthur C, Jaffe RB. Ovarian cancer stem-like side-population cells are tumourigenic and chemoresistant. Br J Cancer 2010; 102: 1276-1283.

16. Gutova M, Najbauer J, Gevorgyan A, Metz MZ, Weng YH, Shih CC et al. Identification of uPAR-positive chemoresistant cells in small cell lung cancer. PLos One 2007; 2: e243.

17. Zhang N, Li R, Tao KS, Cao DY, Ti ZY, Ding R et al. Characterization of a stem-like population in hepatocellular carcinoma MHCC97 cells. Oncol Rep 2010; 23: 827-831.
18. Liu GT, Yuan XP, Zeng ZH, Tunici P, Ng HS, Abdulkadir IR et al. Analysis of gene expression and chemoresistance of $\mathrm{CDI} 33(+)$ cancer stem cells in glioblastoma. Mol Cancer 2006; 5: 67.

19. Adhikari AS, Agarwal N, Wood BM, Porretta C, Ruiz B, Pochampally RR et al. CD117 and Stro-1 identify osteosarcoma tumor-initiating cells associated with metastasis and drug resistance. Cancer Res 2010; 70: 4602-4612.

20. Li C, Heidt DG, Dalerba P, Burant CF, Zhang L, Adsay V et al. Identification of pancreatic cancer stem cells. Cancer Res 2007; 67: 1030-1037.

21. Hasegawa S, Eguchi $\mathrm{H}$, Nagano $\mathrm{H}$, Konno M, Tomimaru $\mathrm{Y}$, Wada $\mathrm{H}$ et al. MicroRNA-1246 expression associated with CCNG2-mediated chemoresistance and stemness in pancreatic cancer. Br J Cancer 2014; 111: 1572-1580.

22. Silverman DT, Swanson CA, Gridley G, Wacholder S, Greenberg RS, Brown LM et al. Dietary and nutritional factors and pancreatic cancer: a case-control study based on direct interviews. J Natl Cancer Inst 1998; 90: 1710-1719.

23. Boots AW, Haenen GR, Bast A. Health effects of quercetin: from antioxidant to nutraceutical. Eur J Pharmacol 2008; 585: 325-337.

24. Kallifatidis G, Rausch V, Baumann B, Apel A, Beckermann BM, Groth et al. Sulforaphane targets pancreatic tumour-initiating cells by NF-kappaB-induced antiapoptotic signalling. Gut 2009; 58: 949-963.

25. Kallifatidis G, Labsch S, Rausch V, Mattern J, Gladkich J, Moldenhauer G et al. Sulforaphane increases drug-mediated cytotoxicity toward cancer stem-like cells of pancreas and prostate. Mol Ther 2011; 19: 188-195.

26. Rausch V, Liu L, Kallifatidis G, Baumann B, Mattern J, Gladkich J et al. Synergistic activity of sorafenib and sulforaphane abolishes pancreatic cancer stem cell characteristics. Cancer Res 2010; 70: 5004-5013.

27. Zhou W, Kallifatidis G, Baumann B, Rausch V, Mattern J, Gladkich J et al. Dietary polypheno quercetin targets pancreatic cancer stem cells. Int J Oncol 2010; 37: 551-561.

28. Appari M, Babu KR, Kaczorowski A, Gross W, Herr I. Sulforaphane, quercetin and catechins complement each other in elimination of advanced pancreatic cancer by miR-let-7 induction and K-ras inhibition. Int J Oncol 2014; 45: 1391-1400.

29. Srivastava RK, Tang SN, Zhu W, Meeker D, Shankar SSulforaphane synergizes with quercetin to inhibit self-renewal capacity of pancreatic cancer stem cellsFront Biosci (Elite Ed) 2011; 3: 515-528.

30. Fan P, Liu L, Yin Y, Zhao Z, Zhang Y, Amponsah PS et al. miR-101-3p reverses gemcitabine resistance by inhibition of ribonucleotide reductase M1 in pancreatic cancer. Cancer Lett 2016; 373: 130-137.

31. Li S, Xie Y, Zhang W, Gao J, Wang M, Zheng G et al. Interferon alpha-inducible protein 27 promotes epithelial-mesenchymal transition and induces ovarian tumorigenicity and stemness. J Surg Res 2015; 193: 255-264.

32. Zhang Z, Liu X, Feng B, Liu N, Wu Q, Han Y et al. STIM1, a direct target of microRNA-185, promotes tumor metastasis and is associated with poor prognosis in colorectal cancer. Oncogene 2015; 34: 4808-4820.

33. Kondratska K, Kondratskyi A, Yassine M, Lemonnier L, Lepage G, Morabito et al. Orai1 and STIM1 mediate SOCE and contribute to apoptotic resistance of pancreatic adenocarcinoma. Biochim Biophys Acta 2014; 1843: 2263-2269.

34. Peche LY, Ladelfa MF, Toledo MF, Mano M, Laiseca JE, Schneider C et al. Human MageB2 protein expression enhances E2F transcriptional activity, cell proliferation, and resistance to ribotoxic stress. J Biol Chem 2015; 290: 29652-29662.

35. Lopez-Ayllon BD, Moncho-Amor V, Abarrategi A, Ibanez de Caceres I, Castro-Carpeno J, Belda-Iniesta $C$ et al. Cancer stem cells and cisplatin-resistant cells isolated from nonsmall-lung cancer cell lines constitute related cell populations. Cancer Med 2014; 3 : 1099-1111.

36. Xue J, Chi Y, Chen Y, Huang S, Ye X, Niu J et al. MiRNA-621 sensitizes breast cancer to chemotherapy by suppressing FBXO11 and enhancing p53 activity. Oncogene 2016; 35: 448-458.

37. Tamm C, Bower N, Anneren C. Regulation of mouse embryonic stem cell self-renewal by a Yes-YAP-TEAD2 signaling pathway downstream of LIF. J Cell Sci 2011; 124: $1136-1144$

38. Hage C, Rausch V, Giese N, Giese T, Schonsiegel F, Labsch S et al. The novel c-Met inhibitor cabozantinib overcomes gemcitabine resistance and stem cell signaling in pancreatic cancer. Cell Death Dis 2013; 4: e627.

39. Nadia Salem Alrawaiq AA. A review of flavonoid quercetin: metabolism, bioactivity and antioxidant properties. Int J PharmTech Res 2014; 6: 933-941.

40. Mulholland PJ, Ferry DR, Anderson D, Hussain SA, Young AM, Cook JE et al. Pre-clinical and clinical study of QC12, a water-soluble, pro-drug of quercetin. Ann Oncol 2001; 12: 245-248.

41. Ferry DR, Smith A, Malkhandi J, Fyfe DW, deTakats PG, Anderson D et al. Phase I clinical trial of the flavonoid quercetin: pharmacokinetics and evidence for in vivo tyrosine kinase inhibition. Clin Cancer Res 1996; 2: 659-668.

42. Kirsh VA, Peters U, Mayne ST, Subar AF, Chatterjee N, Johnson CC et al. Prospective study of fruit and vegetable intake and risk of prostate cancer. J Natl Cancer Inst 2007; 99 : $1200-1209$.

43. Reagan-Shaw S, Nihal M, Ahmad N. Dose translation from animal to human studies revisited. FASEB J 2008; 22: 659-661.

44. Fahey JW, Zhang Y, Talalay P. Broccoli sprouts: an exceptionally rich source of inducers of enzymes that protect against chemical carcinogens. Proc Natl Acad Sci USA 1997; 94: 10367-10372. 
45. Beckermann BM, Kallifatidis G, Groth A, Frommhold D, Apel A, Mattern J et al. VEGF expression by mesenchymal stem cells contributes to angiogenesis in pancreatic carcinoma. Br J Cancer 2008; 99: 622-631.

46. Liu L, Aleksandrowicz E, Fan P, Schonsiegel F, Zhang Y, Sahr H et al. Enrichment of c-Met+ tumorigenic stromal cells of giant cell tumor of bone and targeting by cabozantinib. Cell Death Dis 2014; 5: e1471.

47. Geback T, Schulz MM, Koumoutsakos P, Detmar M. TScratch: a novel and simple software too for automated analysis of monolayer wound healing assays. Biotechniques 2009; 46: 265-274.

48. Moldenhauer G, Momburg F, Moller P, Schwartz R, Hammerling GJ. Epithelium-specific surface glycoprotein of $\mathrm{Mr} 34,000$ is a widely distributed human carcinoma marker. $\mathrm{Br} J$ Cancer 1987; 56: 714-721. (c) (i) Cell Death and Disease is an open-access journal published by Nature Publishing Group. This work is licensed under a Creative Commons Attribution 4.0 International License. The images or other third party material in this article are included in the article's Creative Commons license, unless indicated otherwise in the credit line; if the material is not included under the Creative Commons license, users will need to obtain permission from the license holder to reproduce the material. To view a copy of this license, visit http://creativecommons.org/licenses/by/4.0/

Supplementary Information accompanies this paper on Cell Death and Disease website (http://www.nature.com/cddis) 